\title{
Deutscher Neurodermitis Bund (DNB) e.V.
}

\author{
Th. Schwennesen \\ Deutscher Neurodermitis Bund, Hamburg, Deutschland
}

Der Deutsche Neurodermitis Bund (DNB) e.V. wurde 1986 in Hamburg aus einer Selbsthilfegruppe heraus gegründet. $\mathrm{Zu}$ dieser Zeit war die medizinische Versorgung der an Neurodermitis erkrankten Patienten nicht zufrieden stellend. In den letzten Jahren hat sich das deutlich verbessert. Die Erforschung der Zusammenhänge und die Entwicklung neuer Therapieansätze bzw. Medikamente hat gute Fortschritte in der Neurodermitistherapie gebracht.

Heute muss der Patient Krankheits-Self-Management lernen, d.h. sein Wissen über die Krankheit optimieren, um präventiv mit der Krankheit leben zu können und diese im täglichen Leben beherrschen zu lernen. Hierbei kann eine Selbsthilfeorganisation sehr hilfreich sein, denn die Zeitnot der Behandelnden setzt in diesen Punkten klare Grenzen.

\section{Ziele des DNB}

Das Hauptziel des DNB ist es, die Lebensqualität von Neurodermitispatienten zu verbessern, wo immer es geht. Weitere Ziele des Vereins sind:

- Informationen und Erfahrungen von Betroffenen, aus Wissenschaft und Therapie zur Behandlung und über die Ursachen von Neurodermitis sammeln.

- Diese Informationen den Mitgliedern, der interessierten Öffentlichkeit, der Forschung und der Medizin vermitteln.

- Eine Diskussion aller Behandlungsverfahren und -mittel anregen und fördern.

- Die nichtbetroffene Öffentlichkeit über Neurodermitis und die sekundären Krankheitsfolgen aufklären.

- Die Interessen der Neurodermitispatienten vertreten und eine «Lobby» bilden gegenüber Staat und Gesellschaft, Krankenkassen, Ärzten sowie Einrichtungen des Gesundheitswesens.

- Die Kooperation und das Verständnis zwischen Ärzten, Therapeuten, Behandelnden und den Patienten fördern.

\section{Gut, mehr über den DNB zu wissen}

- Dem DNB steht ein kompetenter, engagierter und motivierter Wissenschaftlicher Beirat zur Seite.

- Der DNB finanziert sich größtenteils über Mitgliedsbeiträge, Spenden und Unterstützung der gesetzlichen Krankenkassen. Private Krankenkassen fühlen sich hier leider nicht verpflichtet.

- Der DNB ist unabhängig, das heißt weder ideell noch finanziell an einen Behandelnden (Arzt, Heilpraktiker, Psychologe), eine Klinik, an ein Unternehmen oder an staatliche Gesundheitseinrichtungen gebunden.

- Kein Behandlungsverfahren, Arznei-, Hilfsmittel oder sonstiges Präparat kann ohne das Einverständnis des DNB damit werben, vom DNB empfohlen zu sein.

- Der DNB weist auf gesundheitspolitische Ungerechtigkeiten hin.

\section{Mitgliedschaften}

\section{Korporatives Mitglied}

Deutscher Paritätischer Wohlfahrtsverband (DPW) e.V., LV Hamburg

Deutsche Dermatologische Gesellschaft (DDG)

\section{Mitglieder}

Die Mitgliederzahl des DNB variiert um 2500. Die zahlenmäBig stärkste Gruppe sind selbst Betroffene (63\% der Mitglieder), dann folgen hautgesunde Eltern mit einem oder mehreren erkrankten Kindern (34\% der Mitglieder). Es gibt aber auch Ausnahmefälle, in denen z.B. Vater, Mutter und Kind(er) alle betroffen sind.

Bei den Mitgliedern überwiegen klar die Frauen (75\%), auch wenn Neurodermitis bei Männern und Frauen gleich häufig auftritt. Im Gegensatz zur verbreiteten Meinung, Neuroder-

\begin{tabular}{ll}
\hline KARGER & ๑ 2004 S. Karger GmbH, Freiburg \\
Fax +49 76145207 14 & Accessible online at: \\
$\begin{array}{l}\text { E-mail Information@Karger.de } \\
\text { www.karger.com }\end{array}$ & www.karger.com/dps
\end{tabular}


mitis sei eine Erkrankung des Kindesalters, haben sich auch genauso viele betroffene Jugendliche und Erwachsene bis etwa 40 Jahre dem DNB angeschlossen, erst darüber nimmt die Häufigkeit ab.

\section{Bundesweite Informationsstellen und Kontakte}

Etwa 35 Selbsthilfegruppen und 120 ehrenamtliche Ansprechpartner des DNB stehen den Betroffenen im gesamten Bundesgebiet zur Seite.

Monatlich führen die Mitarbeiter der Geschäftsstelle über 300 telefonische Beratungsgespräche mit Mitgliedern, aber auch mit Nichtmitgliedern. Hinzu kommen zahlreiche schriftliche, zum Teil sehr spezielle Anfragen von Mitgliedern. Viele Betroffene haben sich auch schon per Fax beim HautFax (0190-252 052, erster Anruf: 1 Seite mit Inhaltsangabe) informiert.

\section{Die Mitgliederzeitschrift HAUTFREUND}

Die Mitgliederzeitschrift HAUTFREUND erscheint 6-mal im Jahr. Die Druckauflage beträgt 13000 Exemplare. HAUT-
FREUND wird von ca. 30000 Lesern gelesen. Die LeserAusgabe-Quote ist durch Mehrfachnutzen in Kliniken und Arztpraxen hoch. Jede HAUTFREUND-Ausgabe wird darüber hinaus an ca. 600 Multiplikatoren unserer Gesellschaft verschickt.

\section{Kommunikation und Mitgliedsantrag}

\section{Postadresse}

Deutscher Neurodermitis Bund e.V.

Spaldingstraße 210, D-20097 Hamburg

Tel. +40 (0)40 2307 44, Fax +49 (0)40 231008

E-mailinfo@dnb-ev.de

DNB online: $w w w . d n b-e v . d e ; w w w . h a u t f r e u n d . d e$

Info-Line

Tel. 01801360360 (max. 4,6 cts/min)

Mo-Fr 8.00-18.00 Uhr

HautFax

24 h Informationen auf Abruf

Fax 0190252052 (0.62 €/min., erste Information:

1 Seite Inhaltsangabe) 